\title{
Diabetes Technology and Therapy in the Pediatric Age Group
}

\author{
David M. Maahs ${ }^{1}$ and Shlomit Shalitin ${ }^{2,3}$
}

\begin{abstract}
Introduction
AOng all the age GROUPS, achieving goal HbA1c while avoiding hypoglycemia $A$ presents perhaps the greatest challenge in the pediatric age group. Recent data from the Type 1 Diabetes (T1D) Exchange report the highest mean HbA1c in children and adolescents with a peak of $9.2 \%$ in 17 -year-olds (1). In contrast, data from European registries suggest that HbA1c has decreased over the past decades $(2,3)$, although mean HbA1c remains well above the goal of $7.5 \%$ established by ISPAD (4) and recently updated by the ADA (5). Data on severe hypoglycemia ( $\mathrm{SH})$ are somewhat more encouraging. Data from Australia (6) and Germany and Austria $(3,7)$ demonstrate a decrease in SH in youth with T1D. Notably, in these datasets the association of $\mathrm{SH}$ with $\mathrm{HbA1c}$ has flattened across the span of HbA1c, contrary to the DCCT data in which SH increased in a curvilinear fashion with lower HbA1c (8).

A number of reasons exist for the relatively poorer outcomes in pediatric as compared to adult T1D patients, including the physiologic changes associated with puberty and the developmental transition from parental focused diabetes self-care to patient self-care, among others. The need for improved diabetes care, combined with the adaptability of younger people to newer technology, has fueled extensive research with diabetes technology in the past year. Although the role of technology in pediatric diabetes care is well established, research specific to this population is required to establish its safety, acceptability, and effectiveness for children.

We conducted a Medline search for articles dealing with the following topics: diabetes technology, insulin pump therapy (CSII), continuous glucose monitoring (CGM), closed-loop systems, and new therapies in T1D relating to the pediatric age group (0-18 years). We focused on key articles that offer some insight into these issues and were published between July 1, 2014, and June 30, 2015.

In the last year, studies reviewed below evaluated the impact of modern technology on improved metabolic control with evidence of marked disparities in insulin treatment method and diabetes outcomes between different ethnic groups. Another study demonstrated that the accuracy of blood glucose meters affects glucose control and hypoglycemia rate. Some of the studies evaluated the implications and advantages of using the real-time continuous glucose monitoring (CGM) that provides 24-hour continuous glucose measurements in pediatric patients with T1D, in children at risk for T1D, and as a tool for evaluation of prediabetes in obese children. In the last year, different prototypes of closed-loop systems were evaluated. Results about the safety and efficacy of these systems in different settings at home and at diabetes camp, and in very young children are reviewed below. Other reviewed studies evaluated the impact of administration of metformin on BMI and insulin sensitivity in adolescents with $\mathrm{T} 1 \mathrm{D}$, and the effect of oral insulin on immune responses in children at high risk for T1D.
\end{abstract}

\footnotetext{
${ }^{1}$ Barbara Davis Center for Childhood Diabetes, University of Colorado, Denver, CO.

${ }^{2}$ Jesse Z and Sara Lea Shafer Institute for Endocrinology and Diabetes, National Center for Childhood Diabetes, Schneider Children's Medical Center of Israel, Petah Tikva, Israel.

${ }^{3}$ Sackler Faculty of Medicine, Tel Aviv University, Tel Aviv, Israel.
} 
Key Articles Reviewed for this Article

Contrasting the clinical care and outcomes of 2,622 children with type 1 diabetes less than 6 years of age in the United States T1D Exchange and German/Austrian DPV registries

Maahs $D M^{1}$, Hermann $J M^{2}$, DuBose $S N^{3}$, Miller $K M^{3}$, Heidtmann $B^{4}$, DiMeglio $L A^{5}$, RamiMerhar $B^{6}$, Beck $R W^{3}$, Schober $E^{6}$, Tamborlane $W V^{7}$, Kapellen $T M^{8}$, Holl $R W^{2}$ for the DPV Initiative and the T1D Exchange Clinic Network

Diabetologia 2014; 57: 1578-85

Racial-ethnic disparities in management and outcomes among children with type 1 diabetes

Willi $S M^{1}$, Miller $K M^{2}$, DiMeglio $L A^{3}$, Klingensmith $G J^{4}$, Simmons $J H^{5}$,

Tamborlane $W V^{6}$, Nadeau $K J^{7}$, Kittelsrud $J M^{8}$, Huckfeldt $P^{9}$, Beck $R W^{2}$,

Lipman $T^{1}, T 1 D$ Exchange Clinic Network

Pediatrics 2015; 135: 424-34

Accuracy of blood glucose meters for self-monitoring affects glucose control and hypoglycemia rate in children and adolescents with type 1 diabetes

Boettcher C, Dost A, Wudy SA, Flechtner-Mors M, Borkenstein M, Schiel R, Weitzel D, Bechtold-Dalla Pozza S, Wolf J, Holl W, German/Austrian Diabetes Prospective

Documentation Initiative

Diabetes Technol Ther 2015; 17: 275-82

Youth and parent measures of self-efficacy for continuous glucose monitoring: survey psychometric properties

Rasbach $L E^{1,2}$, Volkening $L K^{1}$, Markowitz $J T^{l}$, Butler $D A^{l}$, Katz $M L^{1}$, Laffel $L M B^{1}$

Diabetes Technol Ther 2015; 17: 327-34

Early hyperglycemia detected by continuous glucose monitoring in children at risk for type 1 diabetes

Steck AK, Dong F, Taki I, Hoffman M, Klingensmith GJ, Rewers MJ

Diabetes Care 2014; 37: 2031-33

Continuous glucose monitoring and its relationship to hemoglobin A1C and oral glucose tolerance testing in obese and prediabetic youth

Chan $C L^{l}$, Pyle $L^{2}$, Newnes $L^{l}$, Nadeau $K J^{l}$, Zeitler $P S^{l}$, Kelsey $M M^{l}$

J Clin Endocrinol Metab 2015; 100: 902-10

A remote monitoring system for artificial pancreas support is safe, reliable, and user-friendly

Oron T, Farfel A, Muller I, Miller S, Atlas E, Nimri R, Phillip M

Diabetes Technol Ther 2014; 16: 699-705

MD-Logic overnight control for 6 weeks of home use in patients with type 1 diabetes: randomized crossover trial

Nimri $R^{l}$, Muller $I^{1}$, Atlas $E^{1}$, Miller $S^{1}$, Fogel $A^{l}$, Bratina $N^{2}$, Kordonouri $O^{3}$, Battelino $T^{2,4}$, Danne $T^{3}$, Phillip $M^{1,5}$

Diabetes Care 2014; 37: 3025-32

Feasibility of overnight closed loop therapy in young children with type 1 diabetes aged 3-6 years: comparison between diluted and standard insulin strength

Elleri $D^{l}$, Allen $J M^{1}$, Tauschmann $M^{1}$, El-Khairi $R^{l}$, Benitez-Aguirre $P^{1-3}$, Acerini $C L^{l}$, Dunger $D B^{l}$, Hovorka $R^{l}$

BMJ Open Diabetes Res Care 2014; 2: e000040 


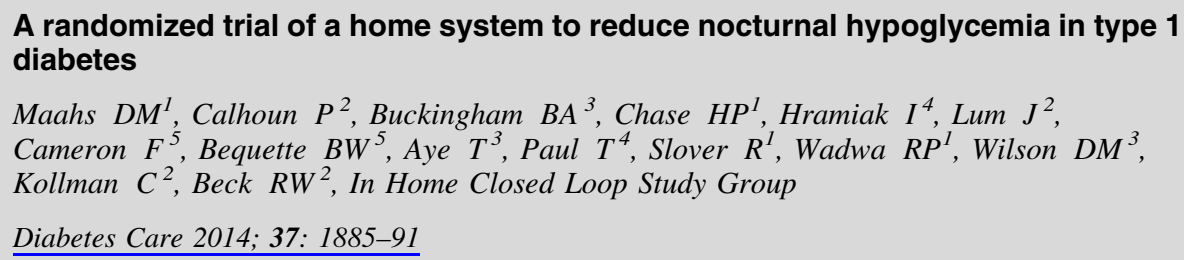

Overnight glucose control with an automated, unified safety system in children and adolescents with type 1 diabetes at diabetes camp

Ly $T T^{1,2}$, Breton $M D^{3}$, Keith-Hynes $P^{3}$, De Salvo $D^{l}$, Clinton $P^{l}$, Benassi $K^{l}$, Mize $B^{3}$, Chernavvsky $D^{3}$, Place $J^{4}$, Wilson $\mathrm{DM}^{1}$, Kovatchev $B P^{3}$, Buckingham $B A^{1}$

Diabetes Care 2014; 37: 2310-16

Effects of high-dose oral insulin on immune responses in children at high risk for type 1 diabetes: the Pre-POINT randomized clinical trial

Bonifacio $E^{1-3}$, Ziegler $A G^{3,4}$, Klingensmith $G^{5}$, Schober $E^{6}$, Bingley $P^{7}$,

Rottenkolber $M^{8}$, Theil $A^{1}$, Eugster $A^{1}$, Puff $R^{4}$, Peplow $C^{4}$, Buettner $F^{9}$, Lange $K^{10}$, Hasford $J^{8}$, Achenbach $P^{3,4}$ for the Pre-POINT Study Group

JAMA 2015; 313: 1541-49

Effects of low dose metformin in adolescents with type 1 diabetes mellitus: a randomized, double-blinded placebo-controlled study

Nadeau KJ, Chow K, Alam S, Lindquist K, Campbell S, McFann K, Klingensmith $G$, Walravens $P$

Pediatr Diabetes 2015; 16: 196-203

\section{Contrasting the clinical care and outcomes of 2,622 children with type 1 diabetes less than 6 years of age in the United States T1D Exchange and German/Austrian DPV registries}

Maahs DM ${ }^{1}$, Hermann $J M^{2}$, DuBose $S N^{3}$, Miller $K M^{3}$, Heidtmann $B^{4}$, DiMeglio $L A^{5}$, RamiMerhar $B^{6}$, Beck $R W^{3}$, Schober $E^{6}$, Tamborlane $W V^{7}$,

Kapellen $T M^{8}$, Holl $R W^{2}$, for the DPV Initiative and the T1D Exchange Clinic Network

${ }^{1}$ Barbara Davis Center for Childhood Diabetes, Aurora, $\mathrm{CO} ;{ }^{2}$ Department of Epidemiology and Medical Biometry, University of Ulm, ZIMBT, Ulm, Germany; ${ }^{3}$ Jaeb Center for Health Research, Tampa, FL; ${ }^{4}$ Catholic Children's Hospital Wilhelmstift Hamburg, Hamburg, Germany; ${ }^{5}$ Department of Endocrinology/Diabetology, Indiana University School of Medicine, Indianapolis, IN; ${ }^{6}$ Department of Pediatrics, Medical University of Vienna, Vienna, Austria; ${ }^{7}$ Department of Paediatric Endocrinology, Yale School of Medicine, Yale University, New Haven, CT; ${ }^{8}$ Women and Children's Centre, University of Leipzig, Leipzig, Germany

Diabetologia 2014; 57: 1578-85

\section{Background}

Children $<6$ years of age present multiple challenges for T1D care. Limited data exist on HbA1c, SH, or DKA outcomes in this age group or whether differences exist between countries. This study aimed to compare participant characteristics, treatment modalities, and clinical outcomes in reg- istry participants in the Type 1 Diabetes Exchange (T1DX) in the United States and the Prospective Diabetes Follow-up Registry (DPV) in Germany and Austria.

\section{Methods}

$\mathrm{HbA} 1 \mathrm{c}, \mathrm{SH}$, and DKA and frequencies of attaining $\mathrm{HbA} 1 \mathrm{c}$ $<7.5 \%$ (ISPAD goal) and < 8.5\% (ADA goal prior to 2014) were compared between registries.

\section{Results}

Insulin pump use was more frequent (74\% vs. 50\%, $p<0.001)$ and HbA1c was lower $(7.4 \%$ vs. $8.2 \%, p<0.001)$ in the DPV compared to the T1DX registry. HbA1c was lower for both pump and injections users in the DPV. Children in the DPV were more likely to achieve the $7.5 \%$ and $8.5 \%$ targets $(56 \%$ vs. $22 \%$ and $90 \%$ vs. $66 \%, p<0.001$ for both). In contrast, $\mathrm{SH}$ did not differ between registries or by HbA1c category $(<7.5 \%, 7.5$ to $<8.5 \%, \leq 8.5 \%)$, whereas DKA increased with HbA1c.

\section{Conclusions}

The DPV data indicate that an HbAlc $<7.5 \%$ can be frequently achieved in children $<6$ years of age with T1D. Improved metabolic control was associated with less DKA without increasing SH. Less frequent use of insulin pumps in the T1DX did not fully explain the suboptimal control in the T1DX, but may relate to the higher HbA1c target previously set by the ADA, which is now harmonized with the ISPAD goal of $<7.5 \%$. 


\section{Comment}

Both the DPV and T1DX registries were established with an objective to improve the care of children with T1D by collecting clinical outcome data on a large population and sharing best practices. Although T1D has increased worldwide in this youngest age group, it remains a relatively rare patient population and requires multicenter collaboration for a sufficient sample size to make comparisons to serve as the basis for improving care. In this article, the U.S. children had higher HbAlc than the German and Austrian children. However, SH did not differ between registries nor did it increase at lower HbAlc (a higher HbAlc to prevent SH had previously been given as a reason for a higher $\mathrm{HbA1c}$ goal). DKA predictably increased with higher HbAlc.

A number of possible explanations for the higher HbAlc are explored, including pump use, which did not fully explain the difference between registries. The higher HbA1c previously set by the ADA is another and in 2014 it was harmonized with the $<7.5 \%$ goal set by ISPAD. Further data are required to determine whether the now lower $\mathrm{HbA} 1 \mathrm{c}$ goal (or other interventions) will translate to improved outcomes in the <6-year-old population in the United States. These data serve as an additional reminder of the power of large registries, including transnational comparisons, to identify shortcomings in diabetes care and identify targets for intervention studies.

Limitations of this study include lack of a central laboratory for $\mathrm{HbAlc}$ measurement, differences in data collection methodology (e.g., the DPV includes 70-90\% of potential patients, whereas the T1DX includes a smaller percentage of potential participants), and the crosssectional nature of the data, which should be considered hypothesis generating. Development of new drugs, devices, and technologies, and strategies to improve care of all children with T1D, including the youngest and most vulnerable, are needed.

\section{Racial-ethnic disparities in management and outcomes among children with type 1 diabetes}

Willi $S M^{1}$, Miller $K M^{2}$, DiMeglio $L A^{3}$, Klingensmith $\mathrm{GJ}^{4}$, Simmons $\mathrm{JH}^{5}$, Tamborlane $\mathrm{WV}^{6}$, Nadeau $\mathrm{KJ}^{7}$, Kittelsrud $J M^{8}$, Huckfeldt $P^{9}$, Beck $R W^{2}$, Lipman $T H^{1}$, T1D Exchange Clinic Network

${ }^{I}$ Children's Hospital of Philadelphia, Philadelphia, PA; ${ }^{2}$ Jaeb Center for Health Research, Tampa, FL; ${ }^{3}$ Indiana University School of Medicine, Indianapolis, IN; ${ }^{4}$ Barbara Davis Center for Childhood Diabetes, University of Colorado, Denver, CO; ${ }^{5}$ Vanderbilt University Medical Center, Nashville, TN; ${ }^{6}$ Department of Paediatric Endocrinology, Yale University, New Haven, CT; ${ }^{7}$ University of Colorado Denver and Children's Hospital Colorado, Denver, CO; ${ }^{8}$ Avera McKennan Hospital and University Health Center, Sioux Falls, SD; ${ }^{9}$ Rand Corporation, Santa Monica, CA

Pediatrics 2015; 135: 424-34

\section{Background}

Racial/ethnic disparities are documented in diabetes treatments and outcomes, although it is controversial whether the disparities are due to socioeconomic status (SES) or other factors. The Type 1 Diabetes Exchange (T1DX) Clinical Registry examined racial/ethnic differences in diabetes therapies and outcomes.

\section{Methods}

The cohort included 10,704 participants $<18$ years with T1D $\geq 1$ year $(8,841$ non-Hispanic whites, 697 non-Hispanic blacks, and 1,166 Hispanic, including 214 high-income [ $>\$ 100,000 /$ year/household] non-Hispanic black and Hispanic families). Diabetes therapies and outcomes were compared.

\section{Results}

Insulin pump use was higher in non-Hispanic white $(61 \%)$ than in non-Hispanic black (26\%) or Hispanic (39\%) participants after adjustment for gender, age, diabetes duration, and SES. Mean HbAlc was higher in non-Hispanic black than in Hispanic or non-Hispanic white participants $(9.6 \%, 8.7 \%, 8.4 \%)$. More non-Hispanic black participants had DKA and severe hypoglycemia in the past year than Hispanic or non-Hispanic white participants who did not differ after adjustment for SES.

\section{Conclusions}

Marked disparities in insulin treatment method and diabetes outcomes, even after SES adjustment, exist between non-Hispanic black versus Hispanic and non-Hispanic white children. Barriers to insulin pump use and optimal glycemic control beyond SES should be explored in all ethnic groups.

\section{Comment}

It has been over 20 years since the publication of the Diabetes Control and Complications Trial demonstrated that intensive management significantly reduced vascular complications of type 1 diabetes (T1D). Data from the T1D Exchange registry indicate that only one quarter of children and adolescents with T1D in the United States achieve the hemoglobin A1c (HbA1c) goal of $<7.5 \%$ (9). Although the importance of tight glucose control is clear, achieving this goal remains elusive in pediatric diabetes.

Willi and the T1D Exchange provide important data documenting disparities in management and outcomes particularly in non-Hispanic black (NHB) children and adolescents with T1D. This large cohort, although not a population representative cohort, included 10,704 participants from 60 pediatric sites in 31 states. The large sample size of this study, including 697 NHB and 1,166 Hispanic participants, enables investigation of factors such as socioeconomic status (SES), parental education, and insurance status that could explain outcome differences (HbAlc, severe hypoglycemia, and diabetic ketoacidosis) by racial-ethnic group. For example, 214 high-income ( $>\$ 100,000)$ NHB families were included, providing sufficient power for stratified analyses by these potential explanatory variables; a limitation of previous reports has been smaller sample sizes.

Higher HbA1c, SH, and DKA in the NHB as compared to non-Hispanic white (NHW) and Hispanic participants were the main findings. Moreover, insulin pump use, 
which is considered by most pediatric endocrinologists to be the best way to deliver insulin, was highest in the NHW population. No significant differences existed between the Hispanic and NHW populations after adjustment for age, gender, T1D duration, and SES, but in the NHB population the disparities in outcomes persisted.

A number of hypotheses to explain higher reported HbA1c in the NHB population have been proposed, including a biologic "glycation gap" between NHW and NHB populations such that for an equivalent mean blood glucose the NHB population has a higher HbA1c than the NHW population. A potentially definitive study to define the association of mean blood glucose with $\mathrm{HbA1c}$ by race-ethnicity is in process by the same T1D Exchange group. Participants will wear a continuous glucose monitor (CGM) over 3 months to measure glucose every 5 minutes. These data will be used to determine whether a difference exists in the relationship of mean CGM glucose with HbA1c between the NHB and NHW populations. While genetic differences may exist in glycation to explain part of the $1.2 \%$ mean difference in HbA1c between NHB and NHW participants reported in this study, it remains to be seen if it will fully explain this disparity.

Cultural, socioeconomic, and provider-related factors may underlie the racial-ethnic disparities in the outcomes. Treatment differences by race-ethnicity in use of insulin pumps (3.6 times higher in NHW than NHB and 1.9 times higher in NHW than Hispanic youth) and selfmonitoring of blood glucose were described. Further research efforts including intervention studies to determine the best possible care for all youth with T1D in the United States are needed.

\section{Accuracy of blood glucose meters for self- monitoring affects glucose control and hypogly- cemia rate in children and adolescents with type 1 diabetes}

Boettcher C, Dost A, Wudy SA, Flechtner-Mors M, Borkenstein M, Schiel R, Weitzel D, Bechtold-Dalla Pozza S, Wolf J, Holl W, German/Austrian Diabetes Prospective Documentation Initiative

Division of Paediatric Endocrinology \& Diabetology, Centre of Child and Adolescent Medicine, Justus Liebig University, Giessen, Germany

Diabetes Technol Ther 2015; 17: 275-82

\section{Background}

Self-monitoring of blood glucose (SMBG) is one of the most important elements of diabetes therapy. To investigate the accuracy of SMBG devices, the standard provided by the International Organization for Standardization (ISO) is widely used. Another method to specify glucose meter performance is via error grids according to Clarke et al. (10) or Parkes et al. (11) that inform the clinician about the severity of errors. The aim of this study was to investigate the accuracy of blood glucose (BG) meters for SMBG and its influence on HbA1c levels and the frequency of hypoglycemic coma.

\section{Methods}

This study is based on the German/Austrian Diabetes software für prospektive Verlaufsbeobachtung (DPV) documentation system, a project that comprises quality assurance and scientific research. Self-measured and simultaneously obtained laboratory BG values from 9,163 patients with T1D younger than 18 years of age were analyzed by investigating their compliance with the ISO criteria (versions 2003 and 2013) and by error grid analyses. Regression models were used to evaluate the effects on glucose control and hypoglycemia rates.

\section{Results}

Comparison of SMBG values with local laboratory results according to the old and new ISO criteria showed that the required $\geq 95 \%$ of $\mathrm{BG}$ values within target were not met under the "real-life conditions"; 78.7-94.7\% of the SMBG values met the old and 79.7-88.6\% met the new ISO criteria. Patients on pump therapy had more outliers than those using injection therapy (old ISO criteria, OR $=1.302,95 \%$ CI 1.084-1.564, $p=0.0051$; new ISO criteria, $\mathrm{OR}=1.155$, 95\% CI 1.011-1.318, $p=0.033$ ). In Clarke and Parkes error grid analyses, the percentages of SMBG values in Zone A ranged between $92.8 \%$ and $94.6 \%$ (Clarke) and between 92.2\% and $95.0 \%$ (Parkes). Logistic regression analysis showed that pump therapy was associated with worse results compared with injection therapy (Clarke, $\mathrm{OR}=1.286$, 95\% CI 1.066-1.551, $p=0.0089$; Parkes, OR $=1.376,95 \%$ CI $1.1481 .650, p=0.0007)$. Age, gender, diabetes duration, and year of treatment had no significant influence on the proportion of BG values within target for both ISO standards, and these parameters were also not related to the proportions of BG values in Zone $\mathrm{A}$ in the error grid analyses. A significant association was found between the deviation of SMBG values from the laboratory-obtained glucose levels and adjusted mean HbA1c levels as a marker of glycemic control: patients measuring "far too low" (Q5) (cutoff, $\geq 0.8 \mathrm{mmol} / \mathrm{L}$ ) with their $\mathrm{SMBG}$ devices showed the highest HbA1c levels (mean, 8.1\% [65 mmol/mol]), differing significantly from Q1 (cutoff, $-0.6 \mathrm{mmol} / \mathrm{L}$ ), Q2 (cutoff, $-0.1 \mathrm{mmol} / \mathrm{L}$ ), Q3 (cutoff, $0.3 \mathrm{mmol} / \mathrm{L}$ ), and Q4 (cutoff, $0.8 \mathrm{mmol} / \mathrm{L}$ ). Those patients who measured "identical or almost identical" (Q3) or "too high" (Q2) exhibited the lowest HbA1c levels (mean, 7.7\% [61 mmol/ mol]). Performing "far too high" was associated with the highest rate of hypoglycemic coma in comparison with the other deviation quintiles.

\section{Conclusions}

This large study showed that under "real-life conditions" the current SMBG devices failed to meet the accuracy criteria of old and new ISO and also failed to meet the desired percentages of Zone A results in Clarke and Parkes error grid analyses. Large deviations of the SMBG values from the "true" glucose levels resulted in higher HbAlc levels and markedly increased rates of hypoglycemic events. 


\section{Comment}

Patients with diabetes and their caregivers expect the available glucose-meter devices to work properly and accurately in order to help them to control their diabetes management. Over the last years several publications have pointed out that not all previously approved glucose-meter devices conform to the current and/or planned ISO standards $(12,13)$, which may have an impact on clinical decision making and glucose control. The current study, which reflects the "real-life conditions" of pediatric patients using their own SMBG devices, emphasizes this issue.

Confounders may contribute to the inaccuracy and interfere with the blood glucose measurements, including lot-to-lot variability of test strips; interference from lack of calibration, temperature, humidity, or poor storage of strips; and patient factors, such as medication and anemia.

With increased use of pump therapy and continuous glucose monitoring (CGM), the use of the pump "bolus calculator" and calibration of the CGM that are based on SMBG values can increase the chance of "inaccurate" values that would consequently lead to inappropriate insulin dose suggestions, with greater deviations from the real required dose of insulin. Patients who measured "far too low" had higher HbA1c values probably because of unnecessary consumption of readily absorbable carbohydrates to raise the blood glucose level that resulted in hyperglycemia and subsequently elevated $\mathrm{HbAlc}$ levels.

A great concern is also for those measuring "too high" with administration of additional unnecessary insulin doses especially in children, placing them at higher risk for hypoglycemia with serious possible sequelae of coma and seizures.

These deviations of SMBG values from the "true" glucose levels lead to a clinically relevant increase of hypoglycemic events with a negative impact on HbAlc levels. Therefore, new stricter international regulations for SMBG devices have to be reconsidered, since patients with diabetes rely on these devices in decision making about their treatment.

\section{Youth and parent measures of self-efficacy for continuous glucose monitoring: survey psychometric properties}

Rasbach $L E^{1,2}$, Volkening $L K^{1}$, Markowitz $J T^{l}$, Butler $D A^{1}$, Katz $M L^{1}$, Laffel $L M B^{1}$

${ }^{1}$ Pediatric, Adolescent, and Young Adult and Genetics and Epidemiology Sections, Joslin Diabetes Center, Harvard Medical School, Boston, MA; ${ }^{2}$ Medical University of South Carolina College of Nursing, Charleston, SC

Diabetes Technol Ther 2015; 17: 327-34

This manuscript is also discussed in the article on: Diabetes Technology and the Human Factor, p. S-105.

\section{Background}

This study aimed to fill a current research gap by developing a psychometric evaluation of youth and parent measures of self-efficacy related to CGM use in pediatric T1D patients.

\section{Methods}

Youth $(n=120)$ with T1D for $\geq 1$ year enrolled in a 2-year randomized clinical trial comparing CGM use with and without the addition of a family-focused CGM behavioral intervention. CGM self-efficacy (CGM-SE) surveys were completed at randomization after a 1-week run in to assess CGM tolerability. Comparisons of predictive validity included the youth in the control arm to assess CGM use and HbAlc outcomes at 3 and 6 months.

\section{Results}

CGM-SE surveys had acceptable internal consistency (Cronbach's $\alpha=0.80$ for youth and 0.82 for parents). Youth with higher baseline CGM-SE ( $>80$ ) had significantly greater CGM use and lower HbA1c after 3 and 6 months compared to those with lower CGM-SE $(\leq 80)$.

\section{Conclusions}

The CGM-SE surveys appear to have strong psychometric properties. CGM self-efficacy may be a tool to assess likelihood of CGM adherence and improvement in glucose control in youth with T1D.

\section{Comment}

A common challenge with new therapies in diabetes is the translation from research to clinical use. Adherence to use of CGM in children with diabetes has been reduced compared to adults in research (14), but similar benefit has been noted in children if CGM are used as directed (15). More recent data indicate that CGM use remains low in the pediatric population in the T1D Exchange at 5-13\% (1), although these data are based on last-generation CGM devices.

Therefore, the challenge remains on how to increase clinical use of CGM so that this technology can benefit the greatest number of children and their families. The CGM-SE tool developed by Laffel and colleagues may be one method to determine whether prospective CGM users are likely to succeed with CGM use. Future work may also include development of programs to improve CGM self-efficacy among those children and parents with low scores so that CGM can be used successfully and more patients can reap its benefits. For the full benefit of diabetes technology to be realized in pediatrics, more studies such as these are required to determine how to best translate technology from research studies to clinical use.

\section{Early hyperglycemia detected by continuous glucose monitoring in children at risk for type 1 diabetes}

Steck AK, Dong F, Taki I, Hoffman M, Klingensmith GJ, Rewers $M J$

Barbara Davis Center for Childhood Diabetes, University of Colorado School of Medicine, Aurora, CO

Diabetes Care 2014; 37: 2031-33 


\section{Background}

CGM was used as a new approach to define early hyperglycemia and to diagnose T1D in children with positive islet antibodies $(\mathrm{Ab})$.

\section{Methods}

A Dexcom SEVEN CGM was worn by $14 \mathrm{Ab}+$ and 9 $\mathrm{Ab}$ - subjects in the Diabetes Autoimmunity Study in the Young (DAISY).

\section{Results}

The Ab + subjects had more hyperglycemia $(>140 \mathrm{mg} /$ dL) than $\mathrm{Ab}$ - subjects (18\% vs. 9\%) with higher maximum daytime glucose values and increased glycemic variability. Mean $\mathrm{HbA1c}$ was $5.5 \%$ in the $\mathrm{Ab}+$ subjects, among whom $\geq 18-20 \%$ CGM time $>140 \mathrm{mg} / \mathrm{dL}$ predicted progression to diabetes.

\section{Conclusions}

CGM can detect early hyperglycemia in $\mathrm{Ab}+$ children at high risk to progress to diabetes. Use of CGM to predict progression of diabetes requires further validation.

\section{Comment}

Current diagnostic tests to predict progression to diabetes include oral glucose tolerance tests (OGTT) and HbAlc measurement. Unfortunately, OGTTs are highly variable and repeat OGTT tests can be a logistical challenge. HbA1c is easier to measure, but less sensitive than OGTTs (16). Therefore, better tests are required to predict who will progress to diabetes. As CGM technology has improved accuracy, reduced burden on the patient, and lowered cost, it presents itself as a possible alternative to OGTTs and HbA1c with an added benefit of obtaining "free-living" glucose data on patients. Further data are required to better understand how to use CGM data to risk stratify progression to diabetes. It should also be noted that these results were obtained with earlier generation CGM technology, which has improved since this study was performed, suggesting that better results are possible in the future.

\section{Continuous glucose monitoring and its relation- ship to hemoglobin A1c and oral glucose tolerance testing in obese and prediabetic youth}

Chan $C L^{1}$, Pyle $L^{2}$, Newnes $L^{1}$, Nadeau $K J^{1}$, Zeitler $P S^{1}$, Kelsey $M M^{1}$

${ }^{1}$ Division of Pediatric Endocrinology, Department of Pediatrics, University of Colorado Denver, Aurora, CO, ${ }^{2}$ Department of Pediatrics, Administrative Division, University of Colorado Denver, Aurora, CO

J Clin Endocrinol Metab 2015; 100: 902-10

\section{Background}

There has been significant controversy over the optimal method of diagnosing prediabetes and type 2 diabetes (T2D) in the pediatric population. Studies in adolescents comparing $\mathrm{HbAlc}$ to 2-hour glucose and fasting plasma glucose (FPG) have reported poor agreement among these tests in classifying youth with dysglycemia, and $\mathrm{HbAlc}$ has been reported to have low sensitivity for identifying prediabetes and diabetes, as defined by oral glucose tolerance test (OGTT). The aim of this study was to evaluate whether HbA1c or the OGTT is a better predictor of "free-living" glucose levels as measured by continuous glucose monitoring (CGM).

\section{Methods}

A cross-sectional study of youth 10-18 years old, body mass index $(\mathrm{BMI}) \geq 85$ th percentile, with diabetes risk factors, not on medications for glucose management.

The participants $(n=118)$ were recruited from primary care and pediatric endocrinology clinics around Denver, Colorado. HbA1c, FPG, and 2-hour glucose were collected and all participants wore a blinded iPro CGM for 72 hours. The ability of $\mathrm{HbAlc}$ and 2-hour glucose to predict abnormal CGM area under the curve (AUC) was evaluated.

Patients with normal glucose levels (HbA1c $<5.7 \%$, FPG $<100 \mathrm{mg} / \mathrm{dL}, 2$-hour glucose $<140 \mathrm{mg} / \mathrm{dL}$ ) were selected, and mean and SD CGM AUC were calculated. The remaining subjects were classified as having an abnormal CGM AUC if their AUC was greater than mean 2 SDs of the normal subjects. Logistic regression models were used to assess the ability of HbA1c, FPG, and 2-hour glucose to predict an abnormal CGM AUC by comparing the area under the receiver operating characteristic (ROC) curves. The cutoff value for HbA1c and 2-hour glucose that maximized sensitivity and specificity for identifying abnormal CGM AUC was determined.

\section{Results}

CGM data of 48 hours were collected on 98 obese youth. There were no differences in age, Tanner staging, or lipids among those with normal or prediabetes HbAlc. There were significant differences between normal glycemic youth and youth with prediabetes for CGM outcomes. When categorized by $\mathrm{HbAlc}$, differences were highly significant $(p<0.0001)$ for night average, night peak glucose, and night AUC, but not for CGM SD or excursions $>200 \mathrm{mg} / \mathrm{dL}$. When categorized by 2 -hour glucose, differences were highly significant ( $p<0.0001$ ) for percentage of time $\geq 140 \mathrm{mg} / \mathrm{dL}$, but not for night-average glucose, minimum-sensor glucose, and night AUC. Participants with prediabetes had on CGM significantly higher average glucose, AUC, peak glucose, and percent of time spent $>120 \mathrm{mg} / \mathrm{dL}$ and $>140 \mathrm{mg} / \mathrm{dL}$ $(p<0.01)$ than youth with normal HbA1c or normal OGTT. $\mathrm{HbA1c}$ had a greater magnitude of correlation to CGM average glucose, AUC, and minimum glucose; 2-hour glucose had a greater magnitude of correlation to CGM SD, peak glucose, and percent of time spent $>140 \mathrm{mg} / \mathrm{dL}$ and $>200 \mathrm{mg} /$ dL. There were no differences in the strength comparisons between 2-hour glucose and HbA1c correlations to CGM outcomes.

\section{Conclusions}

These results confirm that obese youth in their "freeliving" environments frequently have elevated glucoses, as 
measured by CGM, and despite normal HbA1c, FPG, and 2hour glucose, they have higher glucose distributions than in normal-weight, healthy individuals. In obese youth, HbA1c and 2-hour glucose performed equally well at predicting "freeliving" glucose levels on CGM, suggesting that both are acceptable tests for dysglycemia screening.

\section{Comment}

We all wish for the gold-standard screening test for predicting prediabetes and diabetes in youth. This study demonstrated that $\mathrm{HbAlc}$ did not outperform 2-hour glucose in predicting abnormal glucose outcomes. $\mathrm{HbA} 1 \mathrm{c}$ correlated more highly to measures of average and overnight glucose, whereas 2-hour glucose correlated more highly to peak glucose and variability. Thus, $\mathrm{HbA} 1 \mathrm{c}$ reflects a measure of average glycemia, whereas 2-hour glucose reflects the response to a glucose challenge, potentially each test reflecting different underlying pathologic mechanisms for progression to T2D. Although there were no significant differences between 2-hour glucose and $\mathrm{HbA} 1 \mathrm{c}$ in predicting CGM outcomes, both measures outperformed FPG in predicting several CGM outcomes. FPG has little added value to HbA1c or 2-hour glucose in determining abnormal glycemia under "free-living" circumstances. Thus, prediabetes and diabetes can be defined as states of progressive dysglycemia, as reported in this study.

We can also learn from this study that obese youth in their "free-living" environments frequently have elevated glucoses, as measured by CGM. This "silent" hyperglycemia may contribute to an increased cardiovascular risk. Limitations of this study include the small sample size of individuals with diabetes (a larger sample of individuals with diabetes would have allowed further comparison of CGM trends between individuals with prediabetes vs. diabetes), the limited number of CGM variables that were included, and the relevance of these findings to populations in other countries.

\section{A remote monitoring system for artificial pancreas support is safe, reliable, and user-friendly}

\author{
Oron T, Farfel A, Muller I, Miller S, Atlas E, Nimri R, \\ Phillip $M$
}

Jesse Z. and Sara Lea Shafer Institute for Endocrinology and Diabetes, National Center for Childhood Diabetes, Schneider Children's Medical Center of Israel, Petah-Tikva, Israel

Diabetes Technol Ther 2014; 16: 699-705

This manuscript is also discussed in the article on: Diabetes Technology and the Human Factor, p. S-106.

\section{Background}

The widespread use of the Internet and smartphones has further augmented the development of telemedicine systems aimed at improving glycemic control and patient care. The increasing use of continuous glucose monitoring (CGM) devices, as well as the progress in the development of artificial pancreas (AP) systems, suggests a new role for tele- medicine in the management of diabetes. The MD-Logic AP (MDLAP) algorithm applies fuzzy logic theory mimicking lines of reasoning of diabetes caregivers, in addition to realtime learning abilities and safety modules designed to prevent hypoglycemia episodes and prolonged hyperglycemia episodes. The aim of this study was to evaluate the ability of the remote monitoring and control diabetes management system (MDRS) to function as a reliable, remote monitoring system for future AP at-home studies and, in addition, to assess the MDRS ability to prevent or shorten nocturnal hypoglycemia episodes without the use of an AP at home.

\section{Methods}

In this prospective, case-control, randomized study, patients with type 1 diabetes (T1D, $n=18$ ) aged 8-24 years with a mean $\mathrm{HbA} 1 \mathrm{c}$ level of $7.5 \pm 0.6 \%$ treated nightly over a 2week period by the MDRS were compared with patients treated with sensor-augmented pump (SAP) therapy $(n=19)$. The performance of the MDRS, the number and duration of hypo- or hyperglycemia episodes, and the efficacy of the MDRS in detecting them were analyzed. Parents of the study participants completed the hypoglycemia fear survey, attitude, and satisfaction questionnaires.

\section{Results}

One hundred eighty-seven nights $(80.6 \%)$ were valid for the per-protocol analysis in the MDRS group and 166 nights (71.5\%) were valid in the control group. During the 13 nights of the study, the MDRS provided continuous monitoring with no major malfunctions of the system disrupting the connection between the patients and the control and command (C\&C) center.

There was no difference between the study and control groups in regard to the number of hypoglycemia events and time spent in hypoglycemia, as well as in other parameters of glucose control. Without the use of an AP, the MDRS did not significantly prevent nocturnal hypoglycemia episodes. The overall impression of the MDRS users was favorable.

\section{Conclusions}

The MDRS is an efficient and reliable system that allows continuous monitoring of the patients using it, thus enabling the supervising medical personnel to intervene when needed. Without the use of an AP, the current alarm design of the MDRS did not significantly prevent or shorten nocturnal hypoglycemia episodes. The patients and their parents found the system reliable and user-friendly although having a considerable number of false alarms that were mostly related to the CGM device.

\section{Comment}

The idea of remote monitoring of T1D patients is now becoming a reality owing to the widespread use of CGM devices in conjunction with current telecommunication technology. In this feasibility study, the possibility of using a remote monitoring system that is supervised by medical personnel for everyday use was tested. As the MDRS provides continuous monitoring of the patients and continuously delivers technical data to the $C \& C$ 
center, the technical malfunctions were detectable in real time, thus enabling the supervising personnel to intervene according to specific requirements or needs, predefined conditions, and clinical judgment. The MDRS looks suitable for future AP at-home studies and can provide the additional safety layer that is currently required and may relieve patients' and parents' fears, especially during the nights. In the current study, the inability of the MDRS to prevent the detected hypoglycemia episodes relies on the alarm design of the system and the study protocol. Further studies are required to test the hypothesis that by adjusting the alarms and intervention protocol to an open-loop setting, more hypoglycemia episodes could have been prevented or shortened.

It has become clear that remote monitoring systems such as the MDRS are desired by patients and their caregivers and should be considered for patients treated with SAP therapy or future AP systems. However, we have to remember that the MDRS depends greatly on the performance of the CGM. Thus, development of sensors that are more reliable and accurate are required for such a system to be effective.

\section{MD-Logic overnight control for 6 weeks of home use in patients with type 1 diabetes: randomized crossover trial}

Nimri $R^{1}$, Muller $I^{1}$, Atlas $E^{l}$, Miller $S^{1}$, Fogel $A^{l}$, Bratina $N^{2}$, Kordonouri $O^{3}$, Battelino $T^{2,4}$, Danne $T^{3}$, Phillip $M^{1,5}$

${ }^{1}$ Jesse Z. and Sara Lea Shafer Institute for Endocrinology and Diabetes, National Center for Childhood Diabetes, Schneider Children's Medical Center of Israel, Petah Tikva, Israel; ${ }^{2}$ Department of Pediatric Endocrinology, Diabetes and Metabolism, University Medical Center, University Children's Hospital, Ljubljana, Slovenia; ${ }^{3}$ Diabetes Center for Children and Adolescents, AUF DER BULT, Kinder- und Jugendkrankenhaus, Hannover, Germany; ${ }^{4}$ Faculty of Medicine, University of Ljubljana, Ljubljana, Slovenia; ${ }^{5}$ Sackler Faculty of Medicine, Tel Aviv University, Tel Aviv, Israel

Diabetes Care 2014; 37: 3025-32

This manuscript is also discussed in the article on: Closing the Loop, p. S-36.

\section{Background}

The closed-loop insulin delivery system has been tested in hospital settings for day and night glycemic control in patients with type 1 diabetes (T1D).

The MD-Logic system was designed to have several safety layers and fault detection capabilities to suit the requirements for automated control of overnight insulin delivery. The aim was to evaluate the ability of the MD-Logic system to operate safely and efficiently during "free-life" conditions at home over a period of 6 weeks.

\section{Methods}

Patients with T1D aged $12-43$ years $(n=24)$ with an average $\mathrm{HbAlc} 7.5 \pm 0.8 \%(58.1 \pm 8.4 \mathrm{mmol} / \mathrm{mol})$ were ran- domly assigned to participate in two overnight crossover periods, each period of consecutive nights over 6 weeks at patients' homes. One period was under closed loop and the second under sensor-augmented pump (SAP) therapy. The primary end point was overnight time spent with sensor glucose levels $<70 \mathrm{mg} / \mathrm{dL}(3.9 \mathrm{mmol} / \mathrm{L})$. Secondary end points included the time spent at target range $(70-140 \mathrm{mg} / \mathrm{dL})$ and time spent in relatively high and low glucose levels, $>240$ and $<50 \mathrm{mg} / \mathrm{dL}$, respectively.

\section{Results}

During the study, subjects in the closed-loop arm reduced the time spent at hypoglycemia by a median of $40.2 \%$ as compared with subjects in the SAP arm $(p=0.02)$, while the percentage of time spent within $70-140 \mathrm{mg} / \mathrm{dL}$ (3.9$7.8 \mathrm{mmol} / \mathrm{L})$ increased by a median of $21.8 \%(p=0.003)$. The time spent in relatively high blood glucose $>240 \mathrm{mg} / \mathrm{dL}$ $(13.3 \mathrm{mmol} / \mathrm{L})$ was reduced by a median of $52.2 \%$ $(p=0.001)$. Although fewer total overnight insulin doses were delivered per subject on nights when the closed loop was used compared with SAP nights $(p=0.04)$, the mean overnight glucose level was significantly reduced by a median of $15 \mathrm{mg} / \mathrm{dL}$ ( $p=0.008)$. The number of hypoglycemic events per night was low for both groups, but the mean area under the curve for nocturnal hypoglycemic events was $62.5 \%$ lower in the closed-loop group than in the control group ( $p=0.036)$. All tested parameters of hyperglycemia demonstrated a significant improvement during the closedloop nights. A significant positive relation was found between the mean overnight glucose and the mean daily glucose for both closed-loop and SAP therapy. A significant improvement was found in the percentage of glucose readings within $70-180 \mathrm{mg} / \mathrm{dL}$ during the days after closed loop compared with the days after SAP nights $(p=0.006)$. The average daytime glucose levels were also reduced by a median of $10.0 \mathrm{mg} / \mathrm{dL}(p=0.017)$, while total day insulin doses were lower $(p=0.038)$. No severe adverse events occurred during closed-loop control.

\section{Conclusions}

The MD-Logic automated insulin delivery system proved to be a safe and effective tool for overnight treatment of T1D. Glycemic control with MD-Logic showed significantly reduced nocturnal hypoglycemia, with increased time within range and lower mean glucose levels, when used during longterm real-life conditions in patients' homes.

\section{Comment}

Hypoglycemia remains the major concern and obstacle with respect to methods for open-loop control of nocturnal blood glucose. The overnight closed loop has the potential to mitigate the risk of nocturnal hypoglycemia and has an added value over the low glucose suspension (LGS) in maintaining tighter overnight glycemic control, reducing hyperglycemia, improving glucose variability, and reducing patient fasting blood glucose, and thus may consequently improve the daytime control. It seems that the reduction in the risk of hypoglycemia was accompanied by overall better glycemic control. 
The limitations of the study include the small number of participants, and the inability to determine the long-term impact of the MD-Logic system on $\mathrm{HbA} 1 \mathrm{c}$ and quality of life. Also, since the system was evaluated among teens and adults with T1D who were motivated patients with relatively good glycemic control, it is now necessary to evaluate whether the system can reduce the rate of hypoglycemia among patients who are prone to nocturnal hypoglycemia, for those who are poorly controlled and less motivated, and also in the toddler age.

\section{Feasibility of overnight closed loop therapy in young children with type 1 diabetes aged 3-6 years: comparison between diluted and standard insulin strength}

Elleri $D^{l}$, Allen $J M^{1}$, Tauschmann $M^{1}$, El-Khairi $R^{l}$, Benitez-Aguirre $P^{I-3}$, Acerini $C L^{I}$, Dunger $D B^{l}$, Hovorka $R^{1}$

${ }^{I}$ Department of Paediatrics, Wellcome Trust-MRC Institute of Metabolic Science, University of Cambridge, Cambridge, UK; ${ }^{2}$ Department of Paediatrics, Wellcome Trust-MRC Institute of Metabolic Science, University of Cambridge, Cambridge, UK; ${ }^{3}$ Institute of Endocrinology and Diabetes, The Children's Hospital at Westmead, Sydney, Australia

BMJ Open Diabetes Res Care 2014; 2: e000040

\section{Background}

Closed-loop insulin delivery systems combine glucosesensing and insulin-delivery components to modulate delivery of insulin in a glucose-responsive fashion. The efficacy and safety of closed-loop therapy has been demonstrated in clinical studies performed in adolescents and children older than 6 years. The aim of this feasibility study was to evaluate overnight closed-loop therapy with model predictive control in young children with type 1 diabetes (T1D) and to compare closed loop using diluted vs. standard insulin strength.

\section{Methods}

Children with T1D $(n=11)$ aged 3.75-6.96 years with diabetes duration of $2.2 \pm 1$ years, treated with insulin pump therapy, with $\mathrm{HbA} 1 \mathrm{c}$ level of $60 \pm 14 \mathrm{mmol} / \mathrm{mol}$ were studied at a clinical research facility on two occasions. In random order, participants received closed loop with diluted insulin aspart $(20 \mathrm{IU} / \mathrm{mL})$ or closed loop with standard aspart (100 IU/mL) from 17:00 until 8:00 the following morning. Children consumed an evening meal at 17:00 and an optional bedtime snack identical on both occasions.

Meals and carbohydrate content were chosen by the children and their families based on individual preferences and reflecting usual practice at home. Meals were accompanied by insulin boluses calculated using participants' standard insulin pump bolus calculator settings and premeal fingerstick glucose levels. Basal rates on insulin pump were adjusted every $15 \mathrm{~min}$ as directed by a model-predictive-control algorithm informed by a real-time glucose sensor values. Overnight sensor glucose levels were also recorded at home before each study visit while children maintained their usual exercise and eating patterns and standard insulin pump settings were applied.

\section{Results}

Plasma glucose levels remained within the target range of $70-145 \mathrm{mg} / \mathrm{dL}$ for $72 \%$ and $83 \%$ of the time, respectively, when closed loop with standard insulin strength and closed loop with diluted insulin was applied $(p=0.328)$. No difference was found in the mean plasma glucose concentration $(122 \pm 23$ vs. $122 \pm 24 \mathrm{mg} / \mathrm{dL}, p=0.993)$. A trend toward reduced time spent in hypoglycemia (glucose $<70 \mathrm{mg} / \mathrm{dL}$ ) was observed during closed loop with diluted insulin. Sensorbased assessment indicated significant reduction of time $<63 \mathrm{mg} / \mathrm{dL}(p=0.028)$ and a reduced low blood glucose index $(p=0.026)$. Insulin infusion rates were not different during the two interventions and resulted in similar plasma insulin levels.

No episode of hypoglycemia requiring treatment occurred during either intervention.

One asymptomatic episode of hypoglycemia ( $<63 \mathrm{mg} / \mathrm{dL})$ occurred in one participant during closed loop with diluted insulin as compared with 6 episodes in 5 participants during closed loop with standard insulin $(p=0.09)$. All these episodes resolved with no carbohydrate intake within 15$45 \mathrm{~min}$. All measurements of glycemic variability showed a tendency toward reduced variability during closed loop with diluted insulin, although these differences were not statistically significant.

\section{Conclusions}

Closed-loop therapy using a model predictive control approach can be safely and effectively applied for overnight glucose control in young children aged 3-6 years with T1D. A tendency toward reduced hypoglycemia and reduced glucose variability using diluted insulin was observed.

\section{Comment}

Previous studies have reported the efficacy and safety of closed-loop therapy in adolescents and children older than 6 years (17-20). Younger children are more prone to hypoglycemic seizures especially overnight, and the developing brain appears to be more susceptible to the adverse effects of severe hypoglycemia. High insulin sensitivity, unpredictable food intake, and physical activity complicate insulin dose adjustments in young children with T1D, resulting in large glycemic fluctuations. Infants and young children also require very small doses of insulin that challenge the accuracy of subcutaneous insulin administration with standard concentration. Fear of hypoglycemia is common and is associated with increased psychological burden and reduced quality of life for children and their families, leading to higher than optimal glucose levels as an attempt to prevent hypoglycemia. Therefore, the closed-loop system may be particularly beneficial for this group of preschool children. This study demonstrated attainment of safe and efficacious overnight glucose control during overnight closed loop. 
The novelty of the current study was the implementation of the closed-loop system in the young age group and the use of diluted insulin to enhance the accuracy of delivery of small insulin doses that indeed demonstrated a tendency to reduced hypoglycemia and glycemic variability. However, the results of the study are limited due to the small number of patients included in the study, and need to be explored in larger studies. Also, we need to consider the difficulties related to the use of insulin dilution in daily life, including the need to prepare diluted insulin every 7-14 days and the major danger of dosing errors.

\section{A randomized trial of a home system to reduce nocturnal hypoglycemia in type 1 diabetes}

Maahs DM $M^{1}$, Calhoun $P^{2}$, Buckingham $B A^{3}$, Chase $H P^{1}$, Hramiak $I^{4}$, Lum $J^{2}$, Cameron $F^{5}$, Bequette $B W^{5}$, Aye $T^{3}$, Paul $T^{4}$, Slover $R^{1}$, Wadwa $R P^{1}$, Wilson $D M^{3}$, Kollman $C^{2}$, Beck $R W^{2}$, In Home Closed Loop Study Group

${ }^{1}$ Barbara Davis Center for Childhood Diabetes, Aurora, CO; ${ }^{2}$ Jaeb Center for Health Research, Tampa, FL; ${ }^{3}$ Stanford University, Stanford, CA; ${ }^{4}$ St. Joseph's Health Care, London, Ontario, Canada; ${ }^{5}$ Rensselaer Polytechnic Institute, Troy, NY

Diabetes Care 2014; 37: 1885-91

This manuscript is also discussed in the article on: Closing the Loop, p. S-32-33.

\section{Background}

Overnight hypoglycemia is frequent in people with T1D and can result in seizures, loss of consciousness, or death. It remains a barrier to tight glucose control and is feared by patients with T1D and their families. An in-home trial was performed to determine whether a predictive low glucose suspend (PLGS) algorithm to suspend insulin pump delivery based on CGM glucose would result in reduced overnight hypoglycemia.

\section{Methods}

After a run-in phase, 45 individuals aged 15-45 years with T1D were randomized nightly to either PLGS or control for 42 nights. Proportion of nights with CGM glucose $<60 \mathrm{mg} /$ $\mathrm{dL}$ was the primary outcome.

\section{Results}

Overnight hypoglycemia was reduced by $48 \%$ with PLGS vs. control $(21 \%$ vs. $33 \%$ of nights, $p<0.001)$. Median hypoglycemia AUC was reduced by $81 \%$ and hypoglycemia $>2$ hours was reduced $74 \%$. Overnight time with CGM glucose $>180 \mathrm{mg} / \mathrm{dL}$ and morning blood glucose $>250 \mathrm{mg} / \mathrm{dL}$ did not differ. Morning ketosis was $<1 \%$ in both arms of the study.

\section{Conclusions}

Use of an overnight PLGS system reduces overnight hypoglycemia without an increase in morning hyperglycemia or ketosis.

\section{Comment}

The use of a PLGS system represents the second step in the artificial pancreas roadmap (21). The first step, threshold suspension of insulin with a low CGM glucose, has been commercially available since 2009 in many countries and since 2013 in the United States. A PLGS system should reduce time spent hypoglycemic compared to open loop or a threshold suspend system as it reduces insulin in advance of hypoglycemia instead of waiting for hypoglycemia to occur to stop insulin delivery. This study demonstrates that this is indeed the case as compared to open-loop control and that PLGS can do so without increasing ketosis or morning hyperglycemia. Future studies are required to determine the effect on HbAlc and hypoglycemia of an overnight (or 24 hours a day) PLGS system in longer periods and in younger and older populations. The authors note that no difference in hypoglycemia prevention existed between the control and active nights of the system until 23 hours after activation of the system. Unsurprisingly, the PLGS system is unable to prevent hypoglycemia due to insulin dosed before the system activation. This argues that a PLGS system should be used 24 hours a day for a patient to gain the greatest benefit in hypoglycemia prevention. However, even overnight use, should regulatory agencies approve this first, promises benefit for people with T1D. While diabetes care providers and our patients wait for a fully closed loop to mitigate both hypoglycemia and hyperglycemia, overnight PLGS should provide benefit while more advanced technologies await regulatory approval and commercial development.

\section{Overnight glucose control with an automated, unified safety system in children and adolescents with type 1 diabetes at diabetes camp}

Ly $T T^{1,2}$, Breton $M D^{3}$, Keith-Hynes $P^{3}$, De Salvo $D^{1}$, Clinton $P^{1}$, Benassi $K^{1}$, Mize $B^{3}$, Cherñavvsky $D^{3}$, Place $\mathrm{J}^{4}$, Wilson DM ${ }^{1}$, Kovatchev $B P^{3}$, Buckingham $B A^{1}$

${ }^{1}$ Division of Pediatric Endocrinology and Diabetes, Department of Pediatrics, Stanford University School of Medicine, Stanford, CA; ${ }^{2}$ School of Paediatrics and Child Health, The University of Western Australia, Perth, Western Australia, Australia; ${ }^{3}$ Center for Diabetes Technology, University of Virginia, Charlottesville, VA; ${ }^{4}$ Department of Endocrinology, Diabetes and Nutrition, Montpellier University Hospital, Montpellier, France

Diabetes Care 2014; 37: 2310-16

This manuscript is also discussed in the article on: Closing the Loop, p. S-34.

\section{Background}

The study's objective was to assess the safety and efficacy of an automated unified safety system (USS) in providing overnight closed loop (OCL) in children and adolescents with T1D at diabetes camps.

\section{Methods}

The Diabetes Assistant (DiAs) USS with Dexcom G4 Platinum glucose sensor (Dexcom) and t:slim insulin pump 
(Tandem Diabetes Care). An in-patient study to assess safety was performed in 12 participants followed by the main camp study in 20 who were randomized to either OCL or sensor augmented pump (control condition) nightly over the course of the diabetes camp.

\section{Results}

Participants completed 54 OCL and 52 control nights. In an intent-to-treat analysis, percent time in range $70-150 \mathrm{mg} /$ $\mathrm{dL}$ was $62 \%$ vs. $55 \%(p=0.23)$ for OCL versus control nights. In a per protocol analysis, median percent time in range was $73 \%$ vs. $52 \%$ ( $p=0.037)$ in OCL versus control nights. Less time was spent in hypoglycemia during OCL compared to control nights.

\section{Conclusions}

The DiAs USS algorithm improved time spent in range and reduced nocturnal hypoglycemia in children and adolescents with T1D in a diabetes camp setting.

\section{Comment}

Current FDA guidelines for research on artificial pancreas devices advise progression from highly monitored hospital studies to determine safety with progression next to hotel or camp studies that offer the opportunity to continue to have a high level of safety monitoring but a bridge to activities that patients would have in a home setting. In fact, diabetes camps such as the camp used in this study provide a greater challenge with increased physical activity and often a wider variety of diet than may be encountered in the home setting. Studies such as this by Ly advance the field of study and prepare the way for the pivotal, longer term outpatient studies that will be required for regulatory approval. These data demonstrate a significant reduction in nocturnal hypoglycemia in the OCL versus control when faced with the challenge of increased demands of diabetes camp. Depending on the analysis, more time was also spent in target. The next few years promise results from out-patient studies that are needed to assure safety, improved glucose outcomes, and reduced burden for patients with T1D from artificial pancreas devices.

\section{Effects of high-dose oral insulin on immune responses in children at high risk for type 1 diabetes: the Pre-POINT randomized clinical trial}

Bonifacio $E^{1-3}$, Ziegler $A G^{3,4}$, Klingensmith $G^{5}$, Schober $E^{6}$, Bingley $P^{7}$, Rottenkolber $M^{8}$, Theil $A^{1}$, Eugster $A^{1}$, Puff $R^{4}$, Peplow $C^{4}$, Buettner $F^{9}$, Lange $K^{10}$, Hasford $J^{8}$, Achenbach $P^{3,4}$ for the Pre-POINT Study Group

${ }^{1}$ DFG Center for Regenerative Therapies Dresden, Faculty of Medicine, Technische Universität Dresden, Dresden, Germany; ${ }^{2}$ Paul Langerhans Institute Dresden, German Center for Diabetes Research (DZD), Technische Universität Dresden, Dresden, Germany; ${ }^{3}$ Forschergruppe Diabetes e.V., Neuherberg, Germany; ${ }^{4}$ Institute of Diabetes Research, Helmholtz Zentrum München, and For- schergruppe Diabetes, Klinikum rechts der Isar, Technische Universität München, Munich, Germany; ${ }^{5}$ Barbara Davis Center for Childhood Diabetes, University of Colorado School of Medicine, Aurora, CO; ${ }^{6}$ Department of Pediatrics, Medical University of Vienna, Vienna, Austria; ${ }^{7}$ School of Clinical Sciences, University of Bristol, Bristol, United Kingdom; ${ }^{8}$ Institute for Medical Information Sciences, Biometry, and Epidemiology, Ludwig-MaximiliansUniversitaet, Munich, Germany; ${ }^{9}$ Institute of Computational Biology, Helmholtz Zentrum München, Germany; ${ }^{10} \mathrm{De}$ partment of Medical Psychology, Hannover Medical School, Hannover, Germany

JAMA 2015; 313: 1541-49

This manuscript is also discussed in the article on: Immune intervention for type 1 diabetes. p. S-70.

\section{Background}

Type 1 diabetes (T1D) can be detected in asymptomatic individuals by the presence of islet autoantibodies that develop with a peak incidence at around 1 year of age. Autoantibodies against insulin are the first to appear, followed by the expansion of autoimmunity to other antigens before the onset of diabetes. Interferon- $\gamma$-producing CD $4^{+} \mathrm{T}$ cells directed against proinsulin and $\mathrm{CD} 8^{+} \mathrm{T}$ cells directed against insulin are also found in patients with T1D. The human leukocyte antigen (HLA) class II DR4-DQ8 haplotype is found in most children who develop T1D. Oral administration of insulin reduced the development of diabetes in an animal model, probably by stimulation of immune tolerance with induction of insulin-specific regulatory $\mathrm{T}$ cells. Antigen-specific therapy with insulin before the development of autoantibodies may induce protective immune responses that prevent the emergence of autoimmunity and T1D in genetically at-risk children. The aim of the study was to assess whether oral insulin in children autoantibody-negative, genetically at-risk can induce a potentially tolerogenic protective immune response to an autoantigen without causing adverse effects.

\section{Methods}

The Pre-POINT study was a double-blind, placebocontrolled, dose-escalation, phase $1 / 2$ clinical pilot study conducted at four outpatient sites in Germany, Austria, the United States, and the United Kingdom. In this study, islet autoantibody-negative children $(n=25)$ aged 2-7 years with a family history of T1D and susceptible HLA class II genotypes were enrolled. Children were randomized to receive oral insulin $(n=15)$ or placebo $(n=10)$ once daily for $3-18$ months. Children in the insulin group were randomized to receive insulin in 1 of 5 study blocks. Children in block 1 received $2.5 \mathrm{mg}$ of insulin for 6 months, followed by $7.5 \mathrm{mg}$ for 3-12 months; those in block 2 received $2.5 \mathrm{mg}$ for 6 months, followed by $22.5 \mathrm{mg}$ for 3-12 months; those in block 3 received $7.5 \mathrm{mg}$ for 6 months, followed by $67.5 \mathrm{mg}$ for 3-12 months; those in block 4 received $22.5 \mathrm{mg}$ for 3-12 months; and those in block 5 received $67.5 \mathrm{mg}$ for $3-12$ months. The main outcomes were an immune response to insulin, measured as serum IgG and saliva IgA binding to insulin, and CD4 + T-cell proliferative responses to insulin.

A secondary outcome measure was the gene expression profile of $\mathrm{CD}^{+}{ }^{+} \mathrm{T}$ cells responding to insulin. 


\section{Results}

Increases in $\mathrm{IgG}$ binding to insulin, saliva IgA binding to insulin, or $\mathrm{CD}^{+}$T-cell proliferative responses to insulin were observed in 2 of $10(20 \%)$ placebo-treated children and in 1 of $6(16.7 \%)$ children treated with $2.5 \mathrm{mg}$ of insulin, 1 of $6(16.7 \%)$ treated with $7.5 \mathrm{mg}, 2$ of $6(33.3 \%)$ treated with $22.5 \mathrm{mg}$, and 5 of $6(83.3 \%)$ treated with $67.5 \mathrm{mg}(p=0.02)$. Insulin-responsive $\mathrm{T}$ cells displayed regulatory $\mathrm{T}$-cell features after oral insulin treatment. No hypoglycemia, IgE responses to insulin, autoantibodies to glutamic acid decarboxylase or insulinoma-associated antigen 2, or diabetes were observed. Analysis of the gene expression profiles under insulin-stimulated and proinsulin-stimulated proliferation of single-cell-sorted $\mathrm{CD}^{+}{ }^{+} \mathrm{T}$ cells from insulintreated children showed a cluster of cells that expressed FOXP3 (forkhead box P3) without CD127 or cytokines (FOXP3 signature cells), a typical profile of $\mathrm{FOXP} 3^{+}$regulatory T cells. Analysis of the quantitative gene expression data from all genes examined discretely clustered the insulin-responsive $\mathrm{CD}^{+}{ }^{+} \mathrm{T}$ cells from the children who received $67.5 \mathrm{mg}$ of insulin away from the remainder of the insulin-responsive $\mathrm{CD} 4^{+} \mathrm{T}$ cells.

Adverse events were reported in 12 insulin-treated children (67 events) and 10 placebo-treated children (35 events). Infections were the most frequently reported adverse events.

However, there were no signs of hypoglycemia or allergic symptoms after receiving oral insulin. No changes in blood cell counts or blood chemistry values were observed.

\section{Conclusions}

The Pre-POINT pilot study demonstrated that daily oral administration of $67.5 \mathrm{mg}$ of insulin, compared with placebo, to genetically at-risk for T1D healthy children without signs of islet autoimmunity resulted in an immune response without hypoglycemia. The immune response observed in insulin-treated children did not display the features typically associated with T1D, such as a dominant proinflammatory IFNG $\mathrm{CD}^{+}{ }^{+} \mathrm{T}$-cell response.

\section{Comment}

The organ-specific destruction of $\beta$-cell in T1D is mediated by $\mathrm{T}$ helper-1 lymphocyte and develops owing to the interaction between susceptibility genes, environmental factors, and some other risk factors. In order to try to prevent T1D, large multinational investigations have been designed and conducted to evaluate primary prevention trials. Primary prevention is treatment in infants with increased genetic risk before development of symptoms. The primary prevention studies include several dietary manipulations, such as infant formulas free of either cow's milk or bovine insulin, delayed exposure of gluten-containing foods, and vitamin D supplementation. Because primary prevention is directed at individuals who have no clinical signs of autoimmune diseases or metabolic impairment, and because it is uncertain whether they will develop T1D, the designed interventions must be effective, safe, and free of side effects. To date, all primary prevention trials have been dietary interventions designed to interrupt putative environmental factors of T1D. So far, none of the specific dietary factors has been shown to be an unequivocal risk factor for $\beta$-cell-targeted autoimmune diseases or T1D, and their effects have still been contradictory (22).

Secondary prevention is targeted at individuals with persistent islet autoantibodies. In the DPT-1 study, subjects positive for anti-islet cell autoantibodies and anti-insulin autoantibodies without impaired glucose tolerance were randomly allocated to receive oral insulin $7.5 \mathrm{mg}$ per day or placebo (23). The original study demonstrated that there was no delay in the clinical onset of T1D. A post hoc analysis indicated that a considerable delay in the clinical onset of T1D was achieved in a subgroup of individuals with high-titer anti-insulin autoantibodies. A 13-year follow-up also revealed that the $\beta$-cell function was preserved for so long as the oral insulin was taken (24).

In the present study, the administration of oral insulin in children genetically at risk, autoantibody negative, was evaluated as a way of induction of protective immune response to an autoantigen. The T-cell responses to insulin were complementary to the antibody responses at the highest insulin dose. The treatment with oral insulin appeared to induce insulin-responsive and proinsulin-responsive regulatory $\mathrm{T}$ cells, and responses seen with oral insulin were not the dominant proinflammatory interferon- $\gamma$ responses associated with T1D and observed in islet autoantibody-positive children. The findings appear to be consistent with the notion that oral exposure to an antigen can promote regulatory $\mathrm{T}$-cell responses to the antigen. In the study a moderate number of IL21-expressing insulin-responsive $\mathrm{CD} 4^{+} \mathrm{T}$ cells in insulin-treated children was also detected. IL- $21^{+} \mathrm{T}$ cells promote B-cell responses to antigens, and IL21 gene-expressing $\mathrm{CD}^{+}{ }^{+} \mathrm{T}$ cells responding to the vaccine antigen tetanus toxoid were observed, supporting the notion that orally administered insulin acted as a vaccine.

The novelty of the study is that it is the first trial to administer an autoantigen to genetically at-risk children without signs of autoimmunity. However, these children represent less than $1 \%$ of children who ultimately develop T1D, and it is possible that the immune efficacy findings may not be the same in children with lower genetic risk.

This study has other limitations: the small sample size, the short follow-up duration, the enrolled children were older than the peak incidence age of islet autoantibody seroconversion ( 9 months -2 years), and the evidence that insulin doses were not adjusted for body weight. Although the researchers included a combination of assays designed to detect weak responses to determine efficacy, these assays have not been externally validated. Therefore, the findings of immune efficacy should be interpreted with caution.

The findings of this study support the need for larger studies including trials to determine whether oral insulin can prevent islet autoimmunity and progression to diabetes in such children. 


\section{Effects of low dose metformin in adolescents with type 1 diabetes mellitus: a randomized, double- blinded placebo-controlled study}

Nadeau KJ, Chow K, Alam S, Lindquist K, Campbell S, McFann $K$, Klingensmith $G$, Walravens $P$

Division of Pediatric Endocrinology, University of Colorado Denver School of Medicine, Children's Hospital Colorado, Denver, $\mathrm{CO}$

Pediatr Diabetes 2015; 16: 196-203

\section{Background}

Insulin resistance increases in adolescence, complicating glucose control and potentially increasing CVD risk in adolescents with T1D. Metformin is a potential adjunct to insulin therapy in T1D to improve insulin sensitivity and glucose control. The investigators hypothesized that metformin in addition to insulin would improve metabolic parameters in adolescents with T1D.

\section{Methods}

Participants aged $13-20$ years $(n=74)$ were randomized to either metformin or placebo for 6 months. HbA1c, insulin dose, waist circumference, BMI, BP, and fasting lipids were measured.

\section{Results}

Significant decreases in insulin dose, BMI z-score, and waist circumference decreased at 3 and 6 months compared to baseline in the metformin group, and total insulin dose and SBP increased at 3 months and total insulin dose at 6 months in the placebo group. There was no significant change in HbA1c between the metformin or placebo groups.

\section{Conclusions}

Low-dose metformin likely improves BMI and insulin sensitivity in adolescents with T1D. The reduction in waist circumference suggests a benefit on fat distribution. More detailed research, including mechanistic studies, are needed to determine the potential impact of metformin on CVD and glucose control in adolescents with T1DM.

\section{Comment}

The challenge of achieving goal glucose control is nowhere more apparent in adolescents. One physiologic difference is that of pubertal insulin resistance for which increased doses of insulin are required. Metformin is an appealing adjunctive to insulin treatment as a low-cost, safe, and once- or twice-daily oral medication to improve glucose control and CVD risk factors in adolescents with T1D. This study is similar to previous studies indicating improvement in some variables such as total daily insulin dose, BMI, and waist circumference, but not with others such as HbAlc. Perhaps this is not a surprise as the addition of metformin led to a decrease in insulin dose, but not a reduction in mean blood glucose. This was likely because of a lack of intensive dose adjustment to achieve lower blood glucoses as part of the study protocol. Additional multicenter studies are in progress, including hyperinsulinemic-euglycemic clamp studies to investigate the insulin resistance and potential benefits from metformin therapy. Based on present evidence, data do not suggest a sustained improvement in glucose control as measured by $\mathrm{HbA1c}$, but instead potential benefit on CVD risk factors. It remains an open question as to whether these possible benefits are worth initiating metformin therapy in adolescents with T1D or not.

\section{Author Disclosure Statement}

Dr. David Maahs' institute received research funds from Medtronic, Dexcom and Roche. He is on the Scientific Advisory Board for Insulet and a consultant for Abbott.

Dr. Shlomit Shalitin has no competing financial interests.

\section{References}

1. Miller KM, Foster NC, Beck RW, Bergenstal RM, DuBose SN, DiMeglio LA, Maahs DM, Tamborlane WV; T1D Exchange Clinic Network. Current state of type 1 diabetes treatment in the U.S.: updated data from the T1D Exchange Clinic Registry. Diabetes Care 2015; 38: 971-78.

2. Dovc K, Telic SS, Lusa L, Bratanic N, Zerjav-Tansek M, Kotnik P, Stefanija MA, Battelino T, Bratina N. Improved metabolic control in pediatric patients with type 1 diabetes: a nationwide prospective 12-year time trends analysis. Diabetes Technol Ther 2014; 16: 33-40.

3. Rosenbauer J, Dost A, Karges B, Hungele A, Stahl A, Bächle C, Gerstl EM, Kastendieck C, Hofer SE, Holl RW; DPV Initiative and the German BMBF Competence Network Diabetes Mellitus. Improved metabolic control in children and adolescents with type 1 diabetes: a trend analysis using prospective multicenter data from Germany and Austria Diabetes Care 2012; 35: 80-86.

4. Rewers MJ, Pillay K, de Beaufort C, Craig ME, Hanas R, Acerini CL, Maahs DM; International Society for Pediatric and Adolescent Diabetes. ISPAD Clinical Practice Consensus Guidelines 2014. Assessment and monitoring of glycemic control in children and adolescents with diabetes. Pediatr Diabetes 2014; 15 Suppl 20: 102-14.

5. Chiang JL, Kirkman MS, Laffel LM, Peters AL; Type 1 Diabetes Sourcebook Authors. Type 1 diabetes through the life span: a position statement of the American Diabetes Association. Diabetes Care 2014; 37: 2034-54.

6. O'Connell SM, Cooper MN, Bulsara MK, Davis EA, Jones TW. Reducing rates of severe hypoglycemia in a population-based cohort of children and adolescents with type 1 diabetes over the decade 2000-2009. Diabetes Care 2011; 34: 2379-80.

7. Karges B, Rosenbauer J, Kapellen T, Wagner VM, Schober E, Karges W, Holl RW. Hemoglobin A1c levels and risk of severe hypoglycemia in children and young adults with type 1 diabetes from Germany and Austria: a trend analysis in a cohort of 37,539 patients between 1995 and 2012. PLoS Med 2014; 11: e1001742.

8. The Diabetes Control and Complications Trial Research Group. The effect of intensive treatment of diabetes on the development and progression of long-term complications in insulin-dependent diabetes mellitus. N Engl J Med 1993; 329: $977-86$. 
9. Wood JR, Miller KM, Maahs DM, Beck RW, DiMeglio LA, Libman IM, Quinn M, Tamborlane WV, Woerner SE; T1D Exchange Clinic Network. Most youth with type 1 diabetes in the T1D Exchange Clinic Registry do not meet American Diabetes Association or International Society for Pediatric and Adolescent Diabetes clinical guidelines. Diabetes Care 2013; 36: 2035-37.

10. Clarke WL, Cox D, Gonder-Frederick LA, et al. Evaluating clinical accuracy of systems for self-monitoring of blood glucose. Diabetes Care 1987; 10: 622-28.

11. Parkes JL, Slatin SL, Pardo S, et al. A new consensus error grid to evaluate the clinical significance of inaccuracies in the measurement of blood glucose. Diabetes Care 2000; 23: $1143-48$.

12. Brazg RL, Klaff LJ, Parkin CG. Performance variability of seven commonly used self-monitoring of blood glucose systems: clinical considerations for patients and providers. J Diabetes Sci Technol 2013; 7: 144-52.

13. Freckmann G, Schmid C, Baumstark A, et al. System accuracy evaluation of 43 blood glucose monitoring systems for self-monitoring of blood glucose according to DIN EN ISO 15197. J Diabetes Sci Technol 2012; 6: 1060-75.

14. Juvenile Diabetes Research Foundation Continuous Glucose Monitoring Study Group. Continuous glucose monitoring and intensive treatment of type 1 diabetes. $N$ Engl $J$ Med 2008; 359: 1464-76.

15. Juvenile Diabetes Research Foundation Continuous Glucose Monitoring Study Group. Factors predictive of use and of benefit from continuous glucose monitoring in type 1 diabetes. Diabetes Care 2009; 32: 1947-53.

16. Vehik K, Cuthbertson D, Boulware D, Beam CA, Rodriguez H, Legault L, Hyytinen M, Rewers MJ, Schatz DA, Krischer JP; TEDDY, TRIGR, Diabetes Prevention Trial-
Type 1, and Type 1 Diabetes TrialNet Natural History Study Groups. Performance of HbA1c as an early diagnostic indicator of type 1 diabetes in children and youth. Diabetes Care 2012; 35: 1821-25.

17. Phillip M, Battelino T, Atlas E, et al. Nocturnal glucose control with an artificial pancreas at a diabetes camp. $N$ Engl J Med 2013; 368: 824-33.

18. Weinzimer SA, Steil GM, Swan KL, et al. Fully automated closed-loop insulin delivery versus semiautomated hybrid control in pediatric patients with type 1 diabetes using an artificial pancreas. Diabetes Care 2008; 31: 934-39.

19. Elleri D, Allen JM, Kumareswaran K, et al. Closed-loop basal insulin delivery over 36 hours in adolescents with type 1 diabetes: randomized clinical trial. Diabetes Care 2013; 36: 838-44.

20. Hovorka R, Allen JM, Elleri D, et al. Manual closed-loop insulin delivery in children and adolescents with type 1 diabetes: a phase 2 randomised crossover trial. Lancet 2010; 375: 743-51.

21. Kowalski A. Pathway to artificial pancreas systems revisited: moving downstream. Diabetes Care 2015; 38: 1036-43.

22. Knip M, Virtanen SM, Akerblom HK. Infant feeding and the risk of type 1 diabetes. Am J Clin Nutr 2010; 91: $1506 \mathrm{~S}-13 \mathrm{~S}$.

23. Skyler JS, Krischer JP, Wolfsdorf J, et al. Effects of oral insulin in relatives of patients with type 1 diabetes: The Diabetes Prevention Trial-Type 1. Diabetes Care 2005; 28: 1068-76.

24. Vehik K, Cuthbertson D, Ruhlig H, et al. Long-term outcome of individuals treated with oral insulin: diabetes prevention trial-type 1 (DPT-1) oral insulin trial. Diabetes Care 2011; 34: 1585-90. 